\title{
Researchers urge more dementia studies to focus on prevention rather than cure
}

T he prevalence of dementia in western European countries is stabilizing-thanks to societal changes and improved healthcare in the second half of the $20^{\text {th }}$ century-according to a new epidemiological study by Yu-Tzu Wu and colleagues. The multinational team behind the study calls for new healthcare policies that emphasize the importance of preventative research. Their findings are complemented by a new meta-analysis by Jin-Tai Yu and co-workers, which validated several modifiable risk factors for Alzheimer disease $(\mathrm{AD})$ and highlighted the potential for preventative intervention.

The epidemiological study by Wu and co-investigators was initiated to address a lack of recent information on the prevalence of dementia. "In western Europe, epidemiological studies of dementia that were done $20-30$ years ago provided key evidence for dementia policies," explains Wu. "Given the dramatic changes in life expectancy and health profiles between generations, these estimates are now out of date."

The researchers conducted a systematic review of studies that assessed changes in the prevalence and incidence of dementia over time. Owing to a rigorous exclusion criteria that ensured no influence of changes in diagnostic approaches and criteria, they included only five studies in their analysis. Each of these five studies used consistent diagnostic approaches over time to measure true changes in prevalence.

All five studies were population-based cohort studies conducted in different European locations: the UK, Stockholm and Gothenburg in Sweden, Zaragoza in Spain, and Rotterdam in the Netherlands. Each study compared the prevalence (incidence in the Rotterdam study) of dementia in two cohorts that were separated by 7-30 years. The first cohorts were monitored between 1976 and 1989, and the second cohorts were monitored between 1994 and 2008.

The Rotterdam study reported a decrease in the incidence of dementia over a 10-year period. The other four studies reported that the prevalence of dementia either remained stable or reduced over time. These results are not in line with the predictions of escalating prevalence that are based on earlier studies and which drive current healthcare and research policies.

"Our findings do not suggest the expansion of a dementia epidemic, at least within Europe, providing a more optimistic picture than recent public discourse," says Wu. "The stabilization might be related to major investments in these societies in the last century, such as improved education, living conditions and better management of chronic diseases. The observations suggest that reducing the risk of dementia in a whole population is possible."

One strategy to reduce the dementia risk is the identification of modifiable risk factors and early intervention with preventative measures. The meta-analysis of risk factors in AD by Yu and colleagues evaluated 323 studies of potential risk factors for dementia to quantify the level of risk that each of the factors carries.

The analysis identified 11 risk factors with grade I evidence for an association with AD. These factors fell into six categories: pre-existing disease, biochemical, dietary or medical exposures, lifestyle and psychological conditions. The biggest risk factors included smoking, low diastolic blood pressure, a high BMI in midlife and carotid atherosclerosis, all of which increased the risk $>1.5$-fold.

Furthermore, the analysis identified 23 modifiable factors that protect against $\mathrm{AD}$, most of which relate to diet and drug use. "Our study indicates that preventative strategies that target diet, prescription drugs, body chemistry, mental health, underlying disease and lifestyle might help

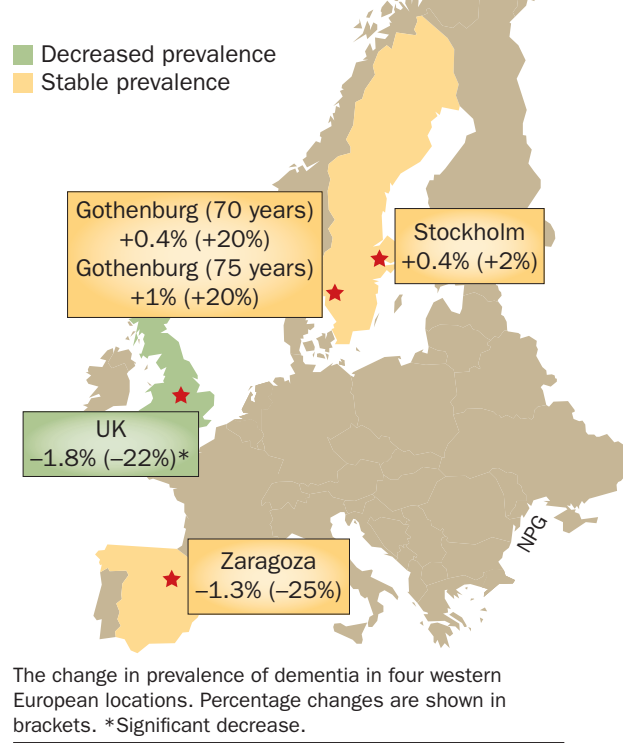

to curb the number of new AD cases," says Yu. "We now need to see significantly more investment into larger prospective cohort studies and clinical prevention trials to provide us with robust evidence about the ways to reduce the risk of AD."

$\mathrm{Wu}$ and colleagues point out in their study that members of the Organisation for Economic Co-operation and Development allocate as little as $3 \%$ of health funding to the prevention of dementia, whereas the majority of funding-for example, $65 \%$ in the UK-goes towards research into disease mechanisms and treatment. As a result, they also call for a strongly asserted balance between investment into population health across the lifecourse and investment into early diagnostics and pharmacological developments, which they say might have a more limited impact.

Ian Fyfe 